\title{
An emergency hierarchical guidance control strategy for autonomous vehicles
}

\author{
Faïza Khelladi, Mohamed Boudali, Rodolfo Orjuela, Mario Cassaro, Michel Basset, and Clément Roos
}

\begin{abstract}
This paper introduces a vehicle guidance control architecture capable of autonomously resolving emergency situations in presence of a steering system failure. The proposed architecture is based on a hierarchical approach composed of three levels, namely the reference generation level, the guidance control level, and the control allocation level. The reference generation first delivers a normal or an emergency trajectory reference and the according speed profile depending on the information sent by the fault detection and isolation (FDI) supervisor. The emergency trajectory allows the vehicle to reach safely the emergency stop strip. The reference trajectory and speed profile tracking is obtained by decoupling the longitudinal and lateral control at a guidance level. Respective errors are subsequently converted into steering and braking/accelerating torques commands. The lowest level of the hierarchy, namely the control allocation, is meant to allocate the control commands to the multiple available actuators according to the FDI information. For example, promoting differential braking when a steering system failure is detected, guaranteeing acceptable tracking performance both in longitudinal and lateral directions.
\end{abstract}

Index Terms-Hierarchical guidance control architecture, trajectory planning, speed profile, lateral guidance, longitudinal guidance, control allocation.

\section{INTRODUCTION}

$\mathbf{O}$ VER the last decades, interest in autonomous vehicles grown to become an intensive research field. As a result, several advanced driver assistance systems (ADAS) have been successfully commercialized like lane centering assist, park assist and adaptive cruise control. These systems are referred to a partial automation (level 2). Nowadays, both academia as well as industry excel to ensure a safe automated driving by exploring the vehicle automation from the conditional automation (level 3) to the full automation (level 5) NHTSA[1].

Many studies start with the highway driving senario since it involves less constraints about the possible obstacle appearance [2], [3], [4], [5], [6], [7]. However, high-speed automated driving still faces problems like the lane change maneuver since it induces a nonlinear vehicle behavior [8], [9].

All studies on the lane change maneuver conducted so far consider that the lateral and the longitudinal motions are ensured by the steering system and the braking/accelerating torques respectively. However, a possible failure of the steering

F. Khelladi, M. Boudali, R. Orjuela, and M. Basset are with the Institut de Recherche en Informatique, Mathématique, Automatique et Signal (IRIMAS EA 7499), Mulhouse, France. e-mail: (faiza-enfel.khelladi, mohamedtaha.boudali, rodolfo.orjuela, michel.basset)@uha.fr

M. Cassaro and C. Roos are with the Office National d'Etudes et de Recherches Aérospatiales (ONERA The French Aerospace Lab), Toulouse, France. e-mail: (mario.cassaro, clement.roos)@onera.fr system (e.g. steer-by-wire) is not taken into consideration. Consequently, the lateral tracking issue can become unrealizable jeopardizing the safety of the system itself. This situation is handled by an emergency guidance requiring a safe stop which seems poorly investigated in the literature.

\section{A. Related works}

For an automated driving development, many fields are explored particularly the perception, the path planning and the control guidance. In fact, these three fields are the needed pillars to build a driverless hierarchical architecture [10], [11] as shown in Fig. 1. The perception level extracts key environmental information as static/dynamic objects' position and road features (e.g. bounds, slopes etc.). These information are then employed by the reference generation level in order to generate a geometric profile for a feasible free obstacles trajectory within the road bounds as well as the associated speed profile. Finally, the control level calculates the appropriate control actions to track the trajectory and the speed references provided by the upstream level.

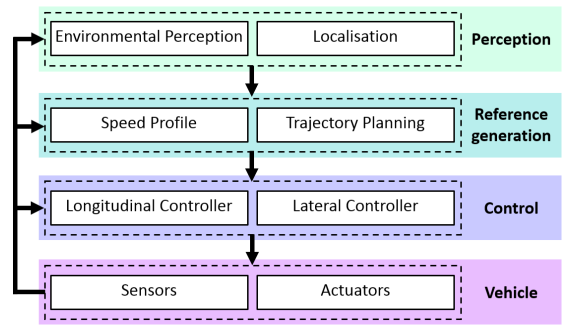

Figure 1: Normal driving strategy for autonomous vehicles

The brief state of the art established below concerns the reference trajectory level and the guidance control level. The perception level is out of the scope of this study. It also concerns only the case of normal driving situation where the vehicle's sensors and actuators are not defective.

The reference generation level regroups two main references, namely the trajectory reference and the speed profile. From the trajectory generation point of view, a wide range of methods have been developed [12], [13]. For instance, the clothoid curves for trajectory planning that suggest a linear change of the curvature is investigated in [8]. The parametric cubic spline for trajectory planning is treated by [14]. In order to have a broader view of the existing trajectory generation methods, the reader can move towards the review of motion planning techniques [12]. The speed profile is closely related 
to the trajectory reference. In fact, the vehicle speed needs to be adjusted according to the road information since it is not static. Hence, it should be increased on straight road and diminished during turns in order to preserve the vehicle's stability [13]. From all the existing approaches, some of them are worth being cited. For example, the speed profile is established according to the friction limits and the stability criteria in [13], [14] and also the comfort criteria in [15], [16].

Using these references, the guidance controller aims to get the appropriate control law that ensures the vehicle reference tracking. The automated guidance requires coupled longitudinal and lateral controllers due to the coupling of the vehicle dynamics [10], [17], [18]. However, this vehicle dynamics complexity makes the controllers design more difficult. A way to get around this problem is to assume a decoupled vehicle dynamics and separately design the longitudinal [19], [20] and the lateral [21], [22], [23] controllers. The listed works show interesting automated guidance results in normal driving conditions even at high speed.

The case of emergency situation seems less investigated in the literature. The main goal in this situation is to ensure the vehicle guidance to safely stop it on the emergency stop strip as illustrated in Fig.2. For that purpose an appropriate reference generation (trajectory planning and speed profile) coupled with a lateral and longitudinal control must be employed.

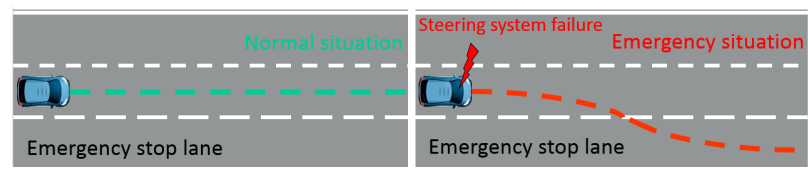

Figure 2: Guidance control objective

A reference generation was recently proposed in [24] based on an optimal control problem formulation. The idea is to define a safe stop references through the minimization of the Euclidean distance between the current position and the final position. This optimization is subject to dynamics constraints (curvature, acceleration,...). A grid of candidate final positions is considered in order to determine the one with a minimal risk (active lane, hard shoulder of the road, emergency stop strip) according to the static/dynamic obstacles and the severity of the internal system fault. However, this approach supposes that all the actuators acting on the lateral/longitudinal dynamics are operational to take control of the vehicle meaning that the steering system failure is not considered.

The emergency guidance when the steering system is defective has been instigated in [25] by replacing the steering action with a guidance moment generated with the help of a differential braking. This approach is based on two high level controllers for the lateral guidance, the first one provides a steering angle during the normal driving situation while the second one provides a yaw moment translated into a differential braking during the emergency situation. In this case, a managing mechanism is proposed to manage the priorities between the longitudinal and the lateral controllers, which use the same actuators (brakes) in the emergency situation. However, the use of two lateral controllers increases the complexity of the guidance controller design.

To overcome this problem, a control architecture using only one lateral control for both situations (normal and emergency) is proposed here. An allocation block is introduced to manage the steering and the differential braking actions.

\section{B. Contributions}

The main contribution in this paper is the proposed guidance control architecture, that takes into account the normal driving and also the emergency driving considered during the steering system failure by using only one high level controller for the lateral guidance. This approach aims to ensure the lateral guidance by using differential braking during the emergency driving in order to achieve a safe stop in the emergency stop strip. Thus, the proposed architecture is composed of the same levels described above (Fig.1) and our focus is on the reference generation and the control levels. Other contributions are highlighted in this paper.

The first contribution is the emergency trajectory reference generation considered as a lane change. This trajectory is based on a geometrical approach by using a hyperbolic tangent, and thus, only few parameters are employed without using an optimization problem formulation.

Another contribution is the speed profile generation during the emergency situation. This speed profile takes into account the trajectory reference information to adjust the deceleration in order to reach safely the emergency stop strip while ensuring the vehicle steerability.

The last contribution concerns the control allocation. This is the lowest level of the architecture, laying between the guidance controller and the vehicle itself, employed to reallocate lateral order consequently to a steering system failure detection. The control allocator objective is to promote differential braking in order to guarantee lateral traking performances and safe vehicle maneuvering.

Throughout this paper, the following notations are used:

- Normal situation: the vehicle's sensors and actuators are not defective.

- Emergency situation: the vehicle's steering system is totally defective.

This paper is organized as follows: Section II presents the vehicle model. The proposed guidance architecture is presented in Section III. In Section IV, the reference generation is detailed. The guidance controller is introduced in Section V. Section VI is dedicated to the control allocation design. The simulation results using the proposed approach are discussed in Section VII.

\section{VEHICLE MODELING}

The 7-DOF vehicle model shown in Fig.3 is used as starting point. The lateral velocity $V_{y}$, the longitudinal velocity $V_{x}$, and the yaw rate $\dot{\psi}$ constitute three degrees of freedom while the wheel velocities $\omega_{i}$ constitute the remaining four degrees of freedom [7]. 
For a 2D references tracking problem, the following assumptions are considered:

- The pitch and the roll actions are neglected.

- The aerodynamic drag force, the rolling resistance force, and the gravitational force are neglected.

- The front wheel steering angle $\delta_{a}$ is assumed to be the same for both front wheels

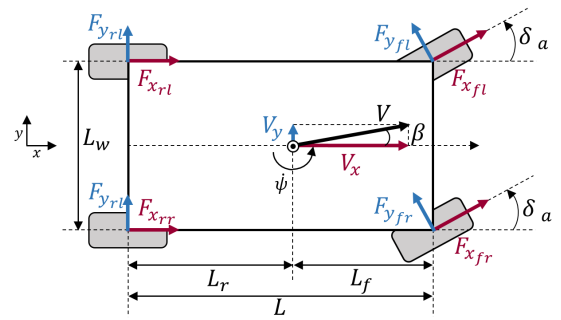

Figure 3: 7-DOF vehicle model

Vehicle dynamics equations. The lateral, the longitudinal, and the yaw motions of the vehicle are respectively given by

$$
\begin{aligned}
m \dot{V}_{x} & =\left(F_{x_{f l}}+F_{x_{f r}}\right) \cos \left(\delta_{a}\right)+F_{x_{r l}}+F_{x_{r r}} \\
& -\left(F_{y_{f l}}+F_{y_{f r}}\right) \sin \left(\delta_{a}\right)+m \dot{\psi} V_{y} \\
m \dot{V}_{y} & =F_{y_{r l}}+F_{y_{r r}}+\left(F_{x_{f l}}+F_{x_{f r}}\right) \sin \left(\delta_{a}\right) \\
& +\left(F_{y_{f l}}+F_{y_{f r}}\right) \cos \left(\delta_{a}\right)-m \dot{\psi} V_{x} \\
I_{z} \ddot{\psi} & =L_{f}\left(F_{y_{f l}}+F_{y_{f r}}\right) \cos \left(\delta_{a}\right)-L_{r}\left(F_{y_{r l}}+F_{y_{r r}}\right) \\
& +\frac{L_{w}}{2}\left(F_{y_{f l}}-F_{y_{f r}}\right) \sin \left(\delta_{a}\right)+M_{z}
\end{aligned}
$$

where $F_{x i}$ and $F_{y i}(i \in\{f l, f r, r l, r r\})$ are the longitudinal and the lateral tire forces at front left, front right, rear left, and rear right respectively, $m$ is the vehicle total mass, $L_{f}$ and $L_{r}$ are the front and rear center of gravity $(\mathrm{CoG})$ distances, $L_{w}$ is the track width, $I_{z}$ is the vehicle moment of inertia around vertical axis. The additional yaw moment $M_{z}$ is defined as

$$
\begin{aligned}
M_{z}= & L_{f}\left(F_{x f l}+F_{x f r}\right) \sin \left(\delta_{a}\right) \\
& +\frac{L_{w}}{2}\left(F_{x f r}-F_{x f l}\right) \cos \left(\delta_{a}\right)+\frac{L_{w}}{2}\left(F_{x r r}-F_{x r l}\right)
\end{aligned}
$$

Wheel dynamics equations. The following torque balance equation is valid for each wheel,

$$
\begin{gathered}
J_{\omega} \dot{\omega}_{i}=T_{i}-r F_{x i} \\
T_{i}=T_{d_{i}}-T_{b_{i}}
\end{gathered}
$$

where $\omega_{i}$ is the wheel velocity, $T_{i}$ the applied wheel torque, $T_{d i}$ the driving torque, $T_{b i}$ the braking torque, $r$ the wheel effective rolling radius and $J_{\omega}$ the wheel moment of inertia.

2-DOF vehicle model. A single-track vehicle model considering small steering angles is used for controllers design. As a result, a linear behavior of the tires can be taken into account [7]

$$
F y_{f, r}=2 C_{f, r} \alpha_{f, r}
$$

where $C_{f}$ and $C_{r}$ are respectively the front and the rear cornering stiffnesses assumed to be constant for a constant friction coefficient. Assuming a small vehicle sideslip angle
( $\left.\beta=\frac{V_{y}}{V_{x}}\right)$, the front and rear slip angles $\alpha_{f}$ and $\alpha_{r}$ can be simplified as

$$
\alpha_{f}=\delta_{a}-\frac{V_{y}+L_{f} \dot{\psi}}{V_{x}}, \quad \alpha_{r}=-\frac{V_{y}-L_{r} \dot{\psi}}{V_{x}}
$$

and using (5) and (6), the lateral dynamics model (1b)-(1c) can be rewritten in a state-space formulation as

$$
\left\{\begin{array}{l}
\dot{x}=A x+B u \\
y=C x+D u
\end{array}\right.
$$

$A=\left[\begin{array}{cc}-\frac{2 C_{f}+2 C_{r}}{m V_{x}} & -\frac{2 C_{f} L_{f}-2 C_{r} L_{r}}{m V_{x}}-V_{x} \\ -\frac{2 C_{f} L_{f}-2 C_{r} L_{r}}{I_{z} V_{x}} & -\frac{2 C_{f} L_{f}^{2}+2 C_{r} L_{r}^{2}}{I_{z} V_{x}}\end{array}\right]$,

$B=\left[\begin{array}{cc}\frac{2 C_{f}}{m} & 0 \\ 0 & \frac{1}{I_{z}}\end{array}\right], C=\mathrm{I}^{2 \times 2}, D=\mathbf{0}^{2 \times 2}$

$x=\left[\begin{array}{c}V_{y} \\ \dot{\psi}\end{array}\right], u=\left[\begin{array}{ll}\delta_{a} & M_{z}\end{array}\right]^{T}$

This model is used for the guidance controller and the control allocation designs described in Sections V and VI.

\section{GUIDANCE CONTROL ARCHITECTURE}

The guidance control objective considered in this study is to safely stop the vehicle on the emergency stop strip during the emergency situation shown in Fig. 2. The performed maneuver consists in leaving the active lane to ensure the passengers safety.

For this purpose, an emergency guidance approach is proposed here. It is based on a hierarchical control architecture presented in Fig.4 and composed of three levels. First, the reference generation provides the geometric reference trajectory and the speed profile for both driving situations (normal and safe stop). Then, the guidance controller is a combination of lateral and longitudinal controllers. The lateral controller gives the appropriate control steering angle, while the longitudinal controller provides the required braking/accelerating torques. Finally, the control allocation commutes the controllers' commands to actuation orders in function of the current driving situation. In this study, it controls the steering system and the four wheel brakes.

The original idea proposed here is to reproduce through the control allocation the same control signals provided by the guidance controller in the normal driving situation. When a failure of the steering system is detected, the control allocation ensures the automated guidance by redistributing the controlled steering input action into a differential braking. This alternative requires a certain minimum speed to guide the vehicle to a standstill since the differential braking strongly affects the longitudinal dynamics. At the same time, the control allocation is able to handle conflicts between the lateral and the longitudinal guidance controllers that use the same actuators in emergency situation.

It is important to note that an FDI block is considered to detect the steering system failure (see [26] and [27]), but its design is out of the scope of this paper. It is supposed that the FDI signal is available and is taken into account at both reference generation and control allocation levels. 


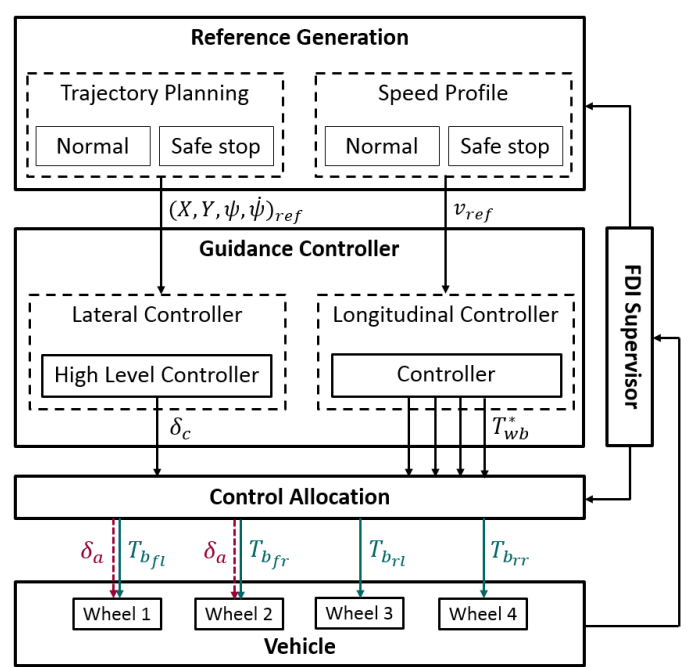

Figure 4: Guidance control architecture

\section{REFERENCE GENERATION}

The reference generation level provides the reference signals needed by the guidance controller. More precisely, it provides the trajectory reference as well as the speed profile that are described in this section.

\section{A. Trajectory planning}

The reference trajectory is composed of four references as shown in Fig.4, namely the reference position $X_{r e f}, Y_{r e f}$, heading $\psi_{\text {ref }}$ and yaw rate $\dot{\psi}_{\text {ref }}$ expressed in a Cartesian coordinate system. The reference trajectory during normal driving is based on the information delivered by the perception module [14]-[13]. Our interest here is focused on the trajectory planning during an emergency situation which consists of a lane change.

The lane change trajectory generation has already been treated in the literature [8], [9], [14]. However, most of the developed methods require many parameters to identify or an expensive optimization in terms of calculation time.

In order to simplify the reference trajectory generation in the emergency situation, the hyperbolic tangent function is used to model mathematically the lane change in the local frame as follows:

$$
\begin{aligned}
& X_{e}(X)=X+a_{1} \\
& Y_{e}(X)=b_{1} \tanh \left(c_{1} X\right)+b_{1}
\end{aligned}
$$

where

$$
\begin{aligned}
\text { where } & =\frac{d_{\text {Long }}}{a_{1}} \\
b_{1} & =\frac{d_{\text {Lat }}}{2} \\
c_{1} & =f\left(\rho_{\max }, b_{1}\right) \\
X & \in\left[-a_{1}, a_{1}\right]
\end{aligned}
$$

Indeed, by using this function, it is quite simple to build the reference trajectory by adjusting only few parameters, namely the lateral and longitudinal gaps $d_{\text {Lat }}$ and $d_{\text {Long }}$ respectively, and the maximal admissible curvature $\rho_{\max }$ as shown in Fig.5. These gaps are obtained according to the expected stopping distances.
Thus, starting from the hyperbolic tangent function that is symmetrical with respect to the origin, $a_{1}$ aims to make a translation along the longitudinal axis, while $b_{1}$ aims to make a translation along the lateral axis and also manage the lateral gap. The path intermediate variable $X$ is used to build the lane change. It is proposed here to compute the parameter $c_{1}$ according to $b_{1}$ and $\rho_{\max }$. In fact, from (8), it is straightforward to compute the reference yaw angle $\psi_{e}$ and path curvature $\rho_{e}$ by using the following expressions [7]

$$
\begin{aligned}
\psi_{e}(X) & =\arctan \left(\frac{d Y_{e}(X)}{d X}\right) \\
\rho_{e}(X) & =\frac{d \psi_{e}(X)}{d X}
\end{aligned}
$$

Hence, the following equations hold

$$
\begin{aligned}
& \psi_{e}(X) \approx \frac{b_{1} c_{1}}{\cosh ^{2}\left(c_{1} X\right)} \\
& \rho_{e}(X)=-\frac{2 b_{1} c_{1}^{2} \sinh \left(c_{1} X\right)}{\cosh ^{3}\left(c_{1} X\right)}
\end{aligned}
$$

The approximation in (10a) is made by assuming small yaw angles due to the large difference between the longitudinal and the lateral gaps $\left(d_{\text {Long }} \gg d_{\text {Lat }}\right)$. The parameter $c_{1}$, in (8b) and (10), aims to manage the shape of the trajectory (soft/tight turning), it is set up by computing the maximal admissible curvature $\rho_{\max }$ at the inflection point

$$
\frac{d \rho_{e}(X)}{d X}=0
$$

From (10b) and (11) it is easy to get $X^{*}$ necessary to compute $\rho_{\max }$ as

$$
\begin{aligned}
& \rho_{\max }=\rho_{e}\left(X^{*}\right) \\
& X^{*}=\frac{\operatorname{asinh}(\sqrt{1 / 2})}{c_{1}}
\end{aligned}
$$

Since $\rho_{\max }$ is one of the input parameters to generate the emergency reference trajectory, (12) aims to get the value of $c_{1}$ using the following expression

$$
c_{1}=\left(\frac{\rho_{\max } \cosh ^{3}\left(d_{1}\right)}{2 b_{1} \sinh \left(d_{1}\right)}\right)^{\frac{1}{2}}
$$

with $d_{1}=\operatorname{asinh}(\sqrt{1 / 2})$.

Depending on the trajectory parameters $\left(d_{\text {Lat }}, d_{\text {Long }}\right.$ and $\left.\rho_{\max }\right)$, some results are shown in Fig.5.

The parameters $d_{\text {Lat }}$ and $d_{\text {Long }}$ should be set up based on the environment information in order to generate a free obstacles trajectory within the road boundaries. Once those parameters have been established, the choice of the maximal path curvature is crucial to get a soft/tight turning as shown in Fig.5. Its value $\rho_{\max }=\frac{\dot{\psi}_{\max }}{V x}$ depends on the vehicle speed $V_{x}$ and the maximum admissible yaw rate $\psi_{\max }$ within the friction limits (which can be determined, see e.g. [7]). Given that the differential braking ensures the lateral guidance during the emergency situation, the maximal path curvature must be small in order to get a soft turning as illustrated in Fig.5. 

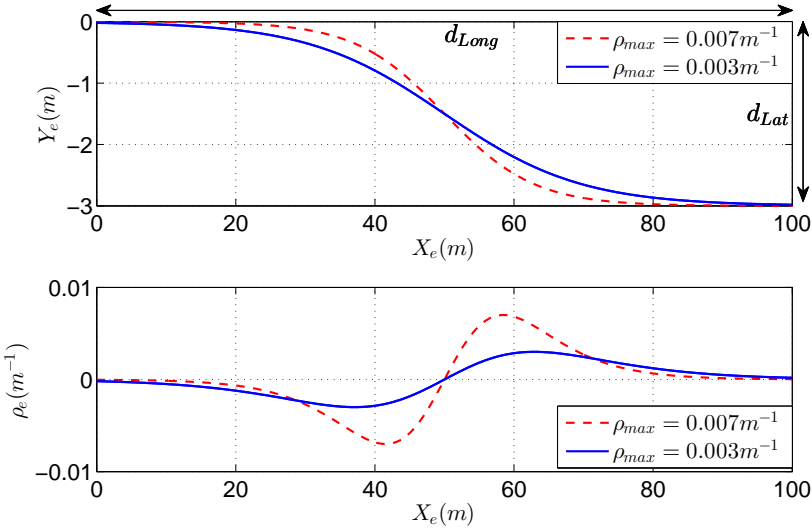

Figure 5: Emergency trajectory generation

Consequently, the brakes are solicited as less as possible in order to respect the tires friction limits. It is assumed that a secure emergency stop strip is available all along the road.

The coordinates (8) are expressed in the vehicle frame system. When a steering system failure is detected, this emergency trajectory is superimposed to the normal reference trajectory expressed in the absolute frame as

$$
\begin{aligned}
& X_{r e f}(X)=X_{e} \cos \left(\psi_{0}\right)-Y_{e} \sin \left(\psi_{0}\right)+X_{0} \\
& Y_{r e f}(X)=X_{e} \sin \left(\psi_{0}\right)+Y_{e} \cos \left(\psi_{0}\right)+Y_{0} \\
& \psi_{\text {ref }}(X)=\psi_{e}+\psi_{0}
\end{aligned}
$$

where $X_{0}, Y_{0}$ and $\psi_{0}$ are the normal reference trajectory coordinates when the failure is detected. Due to the geometrical aspect of the emergency trajectory, this approach is used only when the vehicle is driving in a straight line (highway).

\section{B. Speed profile}

In the autonomous vehicles context, the speed needs to be adjusted according to the road information. In normal driving situation, the velocity should be increased on straight road and reduced during turns in order to preserve the vehicle's stability [13].

Many approaches of the speed profile generation have been developed in the last two decades. The Jerk Limitation method, initially proposed by [15], consists on setting up a jerk function that aims to form acceleration and speed profiles. The goal is to provide these profiles without abrupt changes while respecting the acceleration limits and thus the comfort criteria. In [14] a speed profile, depending on the speed limit, the constrained trajectory, the stability and the driving comfort criteria, was proposed. A quintic Bézier speed profile method was proposed by [16] to improve the comfort by ensuring a smooth Jerk and acceleration profiles. Another speed profile was proposed in [13] considering the tire friction limits to guarantee the feasibility of tracking the path. All theses methods are designed for normal driving situations.

However, only few researches on speed profile generation dealing with an emergency situation that requires a safe stop can be found in the literature. An optimal control problem formulation for references generation, combining speed profile and trajectory reference, during an emergency situation was proposed in [24]. However, this approach does not consider the fact that using differential braking in case of steering system failure strongly influences the longitudinal motion. The variation of the speed profile according to the reference trajectory is also neglected.

In an attempt to remedy this, a finite state machine is used to generate a longitudinal acceleration profile and thus a speed profile. The transition from one state to another depends on the trajectory reference information defined in Section IV-A and more precisely on the paths curvature $\rho_{e}$ and its derivative $\dot{\rho}_{e}$ as shown in Fig.6 and Fig.7.

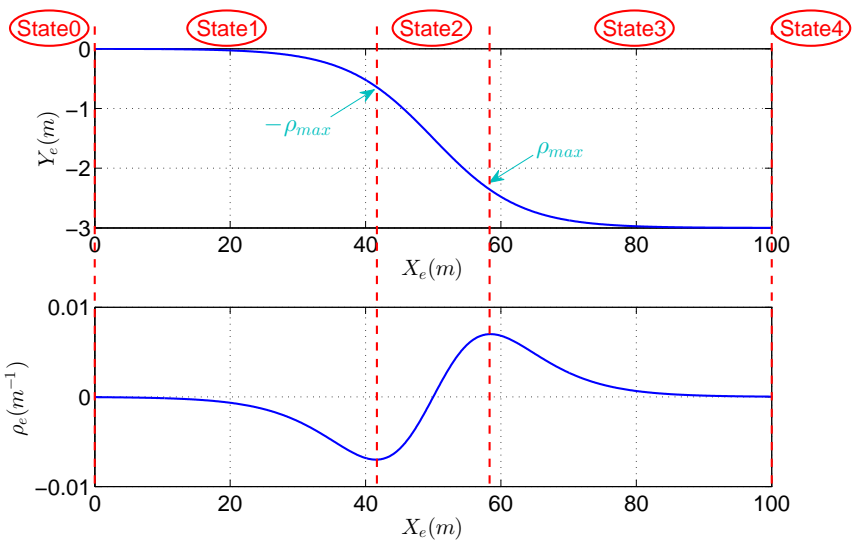

Figure 6: Trajectory and path curvature decomposition

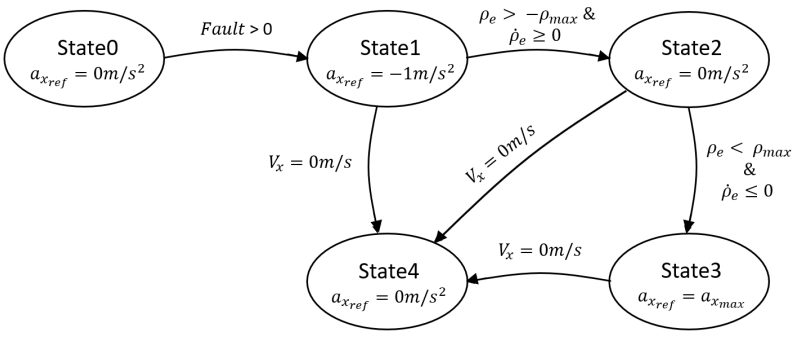

Figure 7: Speed profile generation

It is considered that the reference speed is constant in normal driving (state 0 ). When the failure is detected, the deceleration $a_{x_{r e f}}$ is set at $-1 \mathrm{~m} / \mathrm{s}^{2}$ (state 1), because the differential braking will greatly affect the longitudinal speed which is large at the beginning. When the vehicle is pointing to the right to reach the emergency stop strip, the reference speed is constant in order to maintain the lateral steerability for the rest of the maneuver (state 2). Once the emergency stop strip is reached, the vehicle returns to a straight trajectory and in this case a maximum deceleration $a_{x_{\max }}$ is applied in order to stop it (state 3). When stopped, no acceleration/deceleration is applied (state 4). The resulted acceleration profile of this approach is shown in Fig. 8 when $a_{x_{\max }}=-1.5 \mathrm{~m} / \mathrm{s}^{2}$.

The maximum deceleration in state 3 is set up according to the initial speed $V_{x_{\text {init }}}$ and the stopping distance $d_{\text {Stop }}$, the 


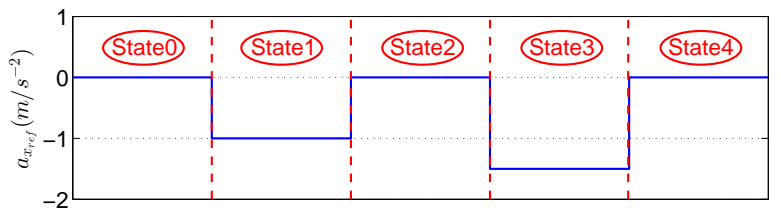

Figure 8: Acceleration profile generation

latter being computed as the difference between $d_{\text {Long }}$ and the value of $X_{e}$ when entering state 3 . The admissible stopping distance according to the initial speed is provided in [28]. By considering a straight motion uniformly decelerated toward $V_{x}=0 \mathrm{~m} / \mathrm{s}$, the following equation indeed holds:

$$
a_{x_{\text {max }}}=-\frac{V_{\text {init }}^{2}}{2 d_{\text {Stop }}}
$$

For instance, if $V_{x_{i n i t}}=30.5 \mathrm{~m} / \mathrm{s}$, the maximum deceleration is shown in Fig.9 as a function of the stopping distance. In fact, for an initial speed already set up, the deceleration is larger when the stopping distance is small.

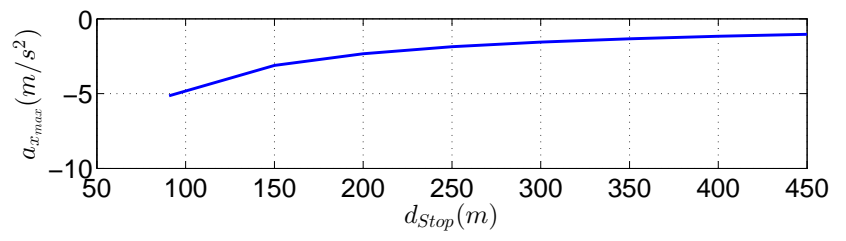

Figure 9: Maximum deceleration according to the stopping distance

\section{GUIDANCE CONTROLLER}

In this section, the guidance controller is presented. As shown in Fig.4, it is composed of a lateral controller tracking the reference trajectory by providing an appropriate steering angle, and a longitudinal controller tracking a reference speed by providing braking or traction torques. These controllers are separately designed.

\section{A. Lateral guidance controller}

The lateral controller goal is to guarantee the reference trajectory tracking and several control laws (PID controller [21], MPC [17], state feedback [7]) have been successfully investigated. These control laws are based on the minimization of the lateral error expressed with respect to the CoG. However, by relying on the CoG, a loss of the path tracking accuracy at the vehicle handling limits can be noticed. To overcome this problem, the lateral error expressed with respect to the center of percussion (CoP) is investigated in [29] as shown in Fig. 10. The advantage of the choice of the $\mathrm{CoP}$ model lies in the fact that the complexity of the lateral dynamics is reduced since the rear lateral force does not affect the motion of the CoP [29].

In this study, the controller synthesis is based on a state feedback control using the CoP model [23]. This model relies on the lateral model described in (7) by considering only the steering angle input. Thus, the CoP model considers the lateral error from the CoP located at a distance of $x_{c o p}$ in the front of the $\mathrm{CoG}$ on the vehicle longitudinal axis as shown in Fig10.

$$
x_{\text {cop }}=\frac{I_{z}}{L_{f} m}
$$

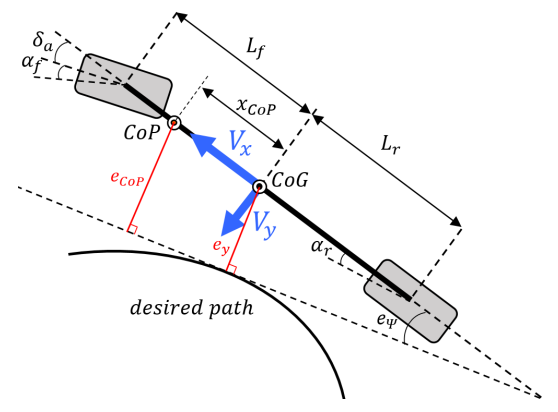

Figure 10: Bicycle model

The CoP error $e_{\text {cop }}$ is expressed according to the CoG error $e_{y}$, the orientation error $e_{\psi}$, and the distance $x_{c o p}$ as follows [23]

$$
e_{c o p}=e_{y}+x_{c o p} e_{\psi}
$$

where,

$$
\dot{e}_{y}=V_{y}+V_{x} e_{\psi}, \quad e_{\psi}=\psi-\psi_{r e f}
$$

with $\psi$ the yaw angle and $\psi_{\text {ref }}$ the desired yaw angle. From (17), it can be noticed that the lateral position error is anticipated and a better tracking trajectory can be expected since the CoP error $e_{\text {cop }}$ is higher than the CoG error $e_{y}$. Using the tracking errors (17) and (18), the CoP model is given by the following state space representation:

$$
\dot{\xi}=A_{c} \xi+B_{c} \delta_{a}+D_{c} d
$$

with $\xi=\left[e_{c o p}, \dot{e}_{c o p}, e_{\psi}, \dot{e}_{\psi}\right]^{T}$ the state vector error, $d=\left[\dot{\psi}_{\text {ref }}, \ddot{\psi}_{\text {ref }}\right]^{T}$ the disturbance vector whose influence should be reduced. The matrices in (19) are:

$$
\begin{aligned}
A_{c} & =\left[\begin{array}{cccc}
0 & 1 & 0 & 0 \\
0 & \frac{-2 R_{l} C_{f}}{m V_{x}} & \frac{2 R_{l} C_{f}}{m} & \frac{2 R_{l} C_{f}\left(x_{c o p}-L_{f}\right)}{m V_{x}} \\
0 & 0 & 0 & 1 \\
0 & \frac{-b}{I_{z} V_{x}} & \frac{b}{I_{z}} & \frac{b x_{c o p}-c}{I_{z} V_{x}}
\end{array}\right], \\
B_{c} & =\left[\begin{array}{c}
0 \\
\frac{2 R_{l} C_{f}}{m} \\
0 \\
\frac{2 C_{f} L_{f}}{I_{z}}
\end{array}\right], D_{c}=\left[\begin{array}{cc}
0 & 0 \\
\frac{-2 R_{l} C_{f} L_{f}}{m V_{x}}-V_{x} & -x_{c o p} \\
0 & 0 \\
\frac{-c}{I_{z} V_{x}} & -1
\end{array}\right],
\end{aligned}
$$

The abbreviations $a, b, c$, and $R_{l}$ are defined as follows

$$
\begin{array}{ll}
a=2\left(C_{f}+C_{r}\right) & b=2\left(L_{f} C_{f}-L_{r} C_{r}\right) \\
R_{l}=\left(L_{f}+L_{r}\right) / L_{r} & c=2\left(L_{f}^{2} C_{f}+L_{r}^{2} C_{r}\right)
\end{array}
$$

The lateral guidance controller design is made by employing the CoP model (19). This controller is based on a feed-forward action $u_{F F}$ coupled to a robust state-feedback $u_{F B}$ described as [23]

$$
\delta_{c}(t)=u_{F F}(t)+u_{F B}(t)
$$

It is worth mentioning that for the controller design, the controller output $\delta_{c}$ is assumed to be applied directly to the 
vehicle actuator $\left(\delta_{c}=\delta_{a}\right)$. When an emergency situation is detected, $\delta_{c}$ will be modified by the control allocation $\left(\delta_{c} \neq \delta_{a}\right)$ as illustrated in Section VI.

Feed-forward control design. The feed-forward action allows to partially reduce the impact of the disturbance $d(t)$ on the state error vector $\xi(t)$. By considering the error $e_{c o p}$ and the matrix $D_{c}$ in (19), the feed-forward action is given by:

$$
u_{F F}=\frac{m}{2 R_{l} C_{f}}\left(\frac{2 R_{l} C_{f} L_{f}}{m V_{x}}+V_{x}\right) \dot{\psi}_{r e f}+\frac{m}{2 R_{l} C_{f}} x_{c o p} \ddot{\psi}_{r e f}
$$

Robust state-feedback control design. The feedback action is the appropriate steering angle that ensures the lateral stability by guaranteeing the exponential convergence to zero of the error vector $\xi(t)$. It also aims to attenuate the impact of the disturbance $d(t)$ on the state variables. The synthesis of this robust controller can be done by using the CoP model (19) and a linear matrix inequality (LMI) based approach as proposed in [23], [25].

$$
u_{F B}=-K_{y} \xi
$$

As previously mentioned, the safe stop maneuver involves a large variation of the vehicle speed. Consequently, it is more relevant for the controller to take into account this variation. To this end, the CoP model (19) can be rewritten under a quasilinear time varying (qLPV) form constituting a set of submodels depending on the vehicle speed $V_{x}$ variation. Hence, the LMI approach for the this qLPV vehicle model can be employed in a straightforward way to design the controller as mentioned in [30].

\section{B. Longitudinal guidance controller}

The longitudinal controller, also called cruise controller (CC), allows to track the speed profile provided by the reference generation module. The longitudinal control systems have been experiencing noteworthy development over the last two decades. For instance, a CC based on a PI controller is proposed in [7] and a sliding mode technique is investigated in [31] for an active CC (ACC). Due to the large variation in the longitudinal velocity for the safe stop maneuver, a nonlinear control based on a direct Lyapunov approach [10] is chosen in this paper in order to cover different vehicle operating points. This controller design depends on the longitudinal model represented by (1a) and (3). Some additional assumptions are considered here:

- The four wheels receive the same torques from the longitudinal controller $\left(T_{f l}=T_{f r}=T_{r l}=T_{r r}=T_{w}\right)$

- A null longitudinal wheel slip is considered, so $r \omega_{i}=V_{x}$. From this assumption combined with the wheel dynamics equation (3) the following expression holds: $F_{x_{i}}=\frac{T_{w}}{r}-$ $\frac{J_{\omega} \dot{V}_{x}}{r^{2}}$

Considering these assumptions, the longitudinal dynamics (1a) can be modeled as

$$
m_{v} \dot{V}_{x}=\frac{4 T_{w}}{r}+m \dot{\psi} V_{y}
$$

where $m_{v}=m+\frac{4 J_{\omega}}{r^{2}}$

It can be noticed that the global torque $T_{w}$ applied on the wheel is the controlled input. A longitudinal controller is used to reduce the speed tracking error

$$
\begin{aligned}
& e_{v}=V_{x_{r e f}}-V_{x} \\
& \dot{e}_{v}=a_{x_{r e f}}-\frac{1}{m_{v}}\left(\frac{4}{r} T_{w}+m \dot{\psi} V_{y}\right)
\end{aligned}
$$

where $V_{x_{r e f}}$ and $a_{x_{r e f}}$ are the speed and the acceleration profiles defined in Section IV-B. Using the Lyapunov approach, the definite positive Lyapunov function is defined as

$$
\begin{aligned}
V & =\frac{1}{2} e_{v}^{2} \\
\dot{V} & =e_{v} \dot{e_{v}}
\end{aligned}
$$

For an exponential convergence toward zero of the tracking error $(24 a)$, the condition below must be verified

$$
\dot{V}=-K_{x} V
$$

with $K_{x}>0$ the decay rate. By introducing (24b) into (25b) and taking into account the stability condition (26), the following control law holds

$$
T_{w}^{*}=\frac{r}{4}\left(m_{v}\left(a_{x_{r e f}}+K_{x} e_{v}\right)-m \dot{\psi} V_{y}\right)
$$

This global torque can be expressed by $T_{w}^{*}=T_{w d}^{*}-T_{w b}^{*}$ as in (4). In this study, it is important to note that only the braking torques $T_{w b}^{*}$ are used during the emergency situation.

Finally, the guidance controller outputs from the lateral and longitudinal controllers $y_{c}$ are expressed as

$$
y_{c}=\left[\begin{array}{lllll}
\delta_{c} & T_{w b}^{*} & T_{w b}^{*} & T_{w b}^{*} & T_{w b}^{*}
\end{array}\right]
$$

\section{CONTROL ALlocAtion}

The automated guidance of vehicles is a reference tracking problem of an over-actuated system composed of the front wheels steering system and the four wheel brakes. The guidance controller outputs $y_{c}$ proposed in Section $\mathrm{V}$ are not directly applied to the vehicle. These signals, in fact, feed the control allocation law and are manipulated, when necessary, according to the FDI information, as shown in Fig.11, before being sent to the actuators.

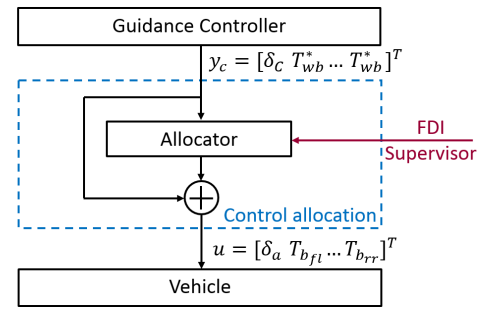

Figure 11: Allocation scheme

During normal driving situations, the allocation outputs $u=$ $\left[\begin{array}{lllll}\delta_{a} & T_{b_{f l}} & T_{b_{f r}} & T_{b_{r l}} & T_{b_{r r}}\end{array}\right]^{T}$ that are applied to the vehicle are simply equal to $y_{c}$ (28). Conversely, during emergency situations, the control allocator penalizes the steering angle $\delta_{c}$ coming from the guidance controller and replace its action by the four wheel brakes action (differential braking). In this case, the applied steering angle $\delta_{a}$ is zero. 
To achieve this task, the allocation theory proposed by [32] is used. This choice of allocator lies on the fact that the allocator dynamics does not affect the vehicle response and thus, it is easy to penalize the steering system without affecting the vehicle behavior.

For the control allocation design, the linear bicycle model (7) is used. By replacing the controlled input $M_{z}$ by its expression (2) and by considering the longitudinal forces as controlled inputs, the matrices $B$ and $D$ can be rewritten as

$$
B=\left[\begin{array}{ccccc}
\frac{2 C_{f}}{m_{f}} & 0 & 0 & 0 & 0 \\
\frac{2 L_{f}}{I_{z}} & \frac{L_{w}}{2 I_{z}} & -\frac{L_{w}}{2 I_{z}} & -\frac{L_{w}}{2 I_{z}} & \frac{L_{w}}{2 I_{z}}
\end{array}\right], \quad D=\mathbf{0}^{2 \times 5}
$$

The controlled inputs (30a) are applied on the wheels as braking torques (30b).

$$
\begin{aligned}
& u^{\prime}=\left[\begin{array}{lllll}
\delta_{a} & F_{x_{f l}} & F_{x_{f r}} & F_{x_{r l}} & F_{x_{r r}}
\end{array}\right]^{T} \\
& T_{i}=r F_{x_{i}} \Longleftrightarrow u=\operatorname{diag}\left[\begin{array}{lllll}
1 & r & r & r & r
\end{array}\right] u^{\prime}
\end{aligned}
$$

As defined in [32], a plant is strongly input-redundant if there is at least one input that can always be replaced by an appropriate combination of the remaining inputs. Thus, the plant inputs must satisfy

$$
\operatorname{Im}\left(B_{\perp}\right)=\operatorname{Ker}\left(\left[\begin{array}{l}
B \\
D
\end{array}\right]\right) \neq \emptyset
$$

where the matrix $B_{\perp}$ satisfies

$$
B_{\perp}^{T} B_{\perp}>0 \text { and }\left[\begin{array}{l}
B \\
D
\end{array}\right] B_{\perp}=0
$$

Verifying this property means guarantee the ability of the allocator to manage the actuators in order to ensure the lateral guidance at all times especially when the steering system is defective. In our case, the vehicle model (7) satisfies this property.

The following dynamic allocator is considered [32]

$$
\begin{aligned}
\dot{w} & =-K B_{\perp}^{T} \bar{W} u \\
u & =y_{c}+B_{\perp} w
\end{aligned}
$$

where $w \in R^{n_{w}}$ is the state vector of the allocator, $u \in R^{n_{u}}$ and $y_{c} \in R^{n_{y}}$ are defined as before. The matrices $K \in$ $R^{n_{w} \times n_{w}}$ and $\bar{W} \in R^{n_{u} \times n_{u}}$ are weighting matrices. $\bar{W}$ is chosen as a diagonal matrix $\bar{W}=\operatorname{diag}\left[\bar{W}_{1} \ldots \bar{W}_{i} \ldots \bar{W}_{n_{u}}\right]$ and has the effect of penalizing or promoting certain actuators action over the others, i.e. the higher the value of $\bar{W}_{i}$, the more the corresponding actuator is penalized. The matrix $K=\lambda I, \lambda \in R^{+}$determines the allocator dynamics and its convergence speed. Effectively, faster response of the allocator can be obtained by employing highest value of $\lambda$, if stability and saturation constraints are met. The goal of the allocator is to inject a signal adjusted by weighting matrices in certain directions of the actuators in order to penalize some of them.

The outcome of the property (32) is that the dynamic of the allocator is invisible from the controller's point of view, so the control allocation does not affect the vehicle states. This result can be deduced by replacing the vehicle input vector $u$ in (7) by its expression from (33). Thus, the allocator being stable by construction (see [32]), the closedloop interconnection with allocation is stable if and only if the closed-loop interconnection without allocation is stable. This is obviously the case here with the guidance controller designed in Section V.

The B matrix (29) shows that only the steering angle acts on the lateral motion of the vehicle. As a result, the steering penalizing issue and thus the reconfiguration of the actuators becomes unrealizable. A solution is to reformulate the mathematical model, in particular the B matrix, accounting for the effect of braking in lateral motion. For this purpose, the moment created around each wheel can be expressed as follows by considering the vehicle model given in Fig.3.

$$
\begin{aligned}
M_{f l} & =\left(L_{f} \sin \left(\delta_{a}\right)-\frac{L_{w}}{2} \cos \left(\delta_{a}\right)\right) F_{x_{f l}} \\
& +\left(L_{f} \cos \left(\delta_{a}\right)+\frac{L_{w}}{2} \sin \left(\delta_{a}\right)\right) F_{y_{f l}} \\
M_{f r} & =\left(L_{f} \sin \left(\delta_{a}\right)+\frac{L_{w}}{2} \cos \left(\delta_{a}\right)\right) F_{x_{f r}} \\
& +\left(L_{f} \cos \left(\delta_{a}\right)-\frac{L_{w}}{2} \sin \left(\delta_{a}\right)\right) F_{y_{f r}} \\
M_{r l} & =-L_{r} F_{x_{r l}}-\frac{L_{w}}{2} F_{y_{r l}} \\
M_{r r} & =-L_{r} F_{x_{r r}}+\frac{L_{w}}{2} F_{y_{r_{r r}}}
\end{aligned}
$$

The sommation of these moments (34a) - (34d) results in the yaw motion equation (1c). Generally, the assumption that the yaw motion is controlled only by the steering angle $\delta_{c}$ provided by the lateral controller is made. As a result, only the lateral forces act on the yaw motion making the longitudinal forces regrouped in (2) acting as a compensation usually used for the lateral stabilization [7]. This assumption can be applied on the equations (34) as follows

$$
\begin{aligned}
\left(L_{f} a_{2}-\frac{L_{w}}{2} b_{2}\right) F_{x_{f l}} & \approx\left(L_{f} b_{2}+\frac{L_{w}}{2} a_{2}\right) F_{y_{f l}} \\
\left(L_{f} a_{2}+\frac{L_{w}}{2} b_{2}\right) F_{x_{f r}} & \approx\left(L_{f} b_{2}-\frac{L_{w}}{2} a_{2}\right) F_{y_{f r}} \\
-L_{r} F_{x_{r l}} & \approx \frac{L_{w}}{2} F_{y_{r l}} \\
-L_{r} F_{x_{r r}} & \approx \frac{L_{w}}{2} F_{y_{r r r}}
\end{aligned}
$$

where $a_{2}=\sin \left(\delta_{a}\right)$ and $b_{2}=\cos \left(\delta_{a}\right)$.

From (35) and with the assumption of a small steering angle, the lateral forces can be expressed in terms of the longitudinal forces as

$$
\begin{aligned}
& F_{y_{f l}} \approx-\frac{L_{w}}{2 L_{f}} F_{x_{f l}} \\
& F_{y_{f r}} \approx \frac{L_{w}}{2 L_{f}} F_{x_{f r}} \\
& F_{y_{r l}} \approx-\frac{L_{w}}{2 L_{r}} F_{x_{r l}} \\
& F_{y_{r r}} \approx \frac{L_{w}}{2 L_{r}} F_{x_{r r}}
\end{aligned}
$$


By using (36a) - (36d), the B matrix (29) becomes

$$
B=\left[\begin{array}{ccccc}
\frac{2 C_{f}}{m} & -\frac{L_{w}}{2 L_{f} m} & \frac{L_{w}}{2 L_{f} m} & -\frac{L_{w}}{2 L_{r} m} & \frac{L_{w}}{2 L_{r} m} \\
\frac{2 C_{f} L_{f}}{I_{z}} & \frac{L_{w}}{2 I_{z}} & -\frac{L_{w}}{2 I_{z}} & -\frac{L_{w}}{2 I_{z}} & \frac{L_{w}}{2 I_{z}}
\end{array}\right]
$$

This B matrix implies that both the steering angle and the tire longitudinal forces can act on the lateral motion of the vehicle.

Considering only braking action on the wheel, the generated force are always negative. This constraint is taken into account, as shown in Fig.12, by using the magnitude saturation expressed by

$$
\operatorname{sat}\left(F_{x i}\right)=\frac{1}{2}+\frac{1}{2} \tanh \left(-\left(\pi+F_{x i}\right)\right)
$$

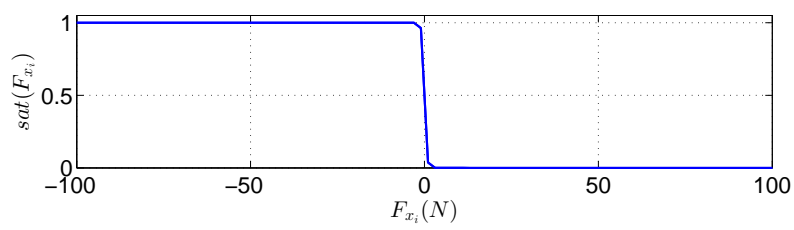

Figure 12: Magnitude saturation of $F_{x_{i}}$

Thereby, the B matrix (39) becomes

$$
B=\left[\begin{array}{ccccc}
\frac{2 C_{f}}{m} & -\frac{L_{w}}{2 L_{f} m} & \frac{L_{w}}{2 L_{f} m} & -\frac{L_{w}}{2 L_{r} m} & \frac{L_{w}}{2 L_{r} m} \\
\frac{2 C_{f} L_{f}}{I_{z}} & \frac{L_{w}}{2 I_{z}} & -\frac{L_{w}}{2 I_{z}} & -\frac{L_{w}}{2 I_{z}} & \frac{L_{w}}{2 I_{z}}
\end{array}\right] \operatorname{sat}\left(F_{x}\right)
$$

where

$\operatorname{sat}\left(F_{x}\right)=\operatorname{diag}\left[1 \operatorname{sat}\left(F_{x f l}\right) \operatorname{sat}\left(F_{x f r}\right) \operatorname{sat}\left(F_{x r l}\right) \operatorname{sat}\left(F_{x r r}\right)\right]$

\section{Simulation RESUlTS}

In this section, the performances of the hierarchical control architecture proposed for emergency situation is investigated. For this purpose, the CarMaker environment is used by considering the vehicle parameters of the Renault Scenic presented in Table I.

\section{A. Test description}

The road case treated here is shown in Fig.13, it corresponds to a turn followed by a straight line in the normal situation (continuous line in Fig.13). Two cases of emergency trajectory are considered according to the emergency parameters described in Table I. In fact, the two cases have the same starting point corresponding to the point when the fault is detected, and the same lateral gap $d_{\text {Lat }}$. However, the longitudinal gap $d_{\text {Long }}$ and thus the maximum path curvature $\rho_{\max }$ differ. It can be noticed that the greater $d_{\text {Long }}$, the smaller $\rho_{\max }$.

During the normal driving situation, the vehicle speed is assumed to be constant $\left(V_{x}=V_{x_{\text {init }}}\right)$. An abrupt complete failure of the steering system is simulated at $t=8 \mathrm{~s}$.

The controller parameters given in Table I are obtained from

\begin{tabular}{|c|c|c|c|}
\hline Parameter & \multicolumn{3}{|l|}{ Value (unit) } \\
\hline \multicolumn{4}{|c|}{ Vehicle parameters } \\
\hline$I_{z}$ & $3503\left(\mathrm{~kg}^{2} \mathrm{~m}\right)$ & $m$ & $1828(\mathrm{~kg})$ \\
\hline$\tilde{J}_{\omega}$ & $0.99\left(\mathrm{~kg}^{2} \mathrm{~m}\right)$ & $C_{f}$ & $97035\left(\mathrm{~N}^{-\mathrm{rad}^{-1}}\right)$ \\
\hline$L_{f}$ & $1.035(\mathrm{~m})$ & $C_{r}$ & $91631\left(\mathrm{~N} \cdot \mathrm{rad}^{-1}\right)$ \\
\hline$L_{r}$ & $1.655(\mathrm{~m})$ & & $0.313(\mathrm{~m})$ \\
\hline$L_{w}$ & $1.535(\mathrm{~m})$ & $V_{x_{i n i t}}$ & $60(\mathrm{~km} / \mathrm{h})$ \\
\hline \multicolumn{4}{|c|}{ Emergency trajectory parameters } \\
\hline \multicolumn{2}{|l|}{ Case 1} & \multicolumn{2}{|l|}{ Case 2} \\
\hline$d_{\text {Lat }}$ & $3.5(\mathrm{~m})$ & $d_{\text {Lat }}$ & $3.5(\mathrm{~m})$ \\
\hline$d_{\text {Long }}$ & $400(\mathrm{~m})$ & $d_{\text {Long }}$ & $200(\mathrm{~m})$ \\
\hline$\rho_{\max }$ & $4 \times 10^{-4}\left(\mathrm{~m}^{-1}\right)$ & $\rho_{\max }$ & $2 \times 10^{-3}\left(\mathrm{~m}^{-1}\right)$ \\
\hline \multicolumn{4}{|c|}{ Controller parameters } \\
\hline$K_{x}$ & \multirow{2}{*}{\multicolumn{3}{|c|}{$\begin{array}{llll}-50 & & & \\
{[0.5133} & 0.0752 & 6.0051 & 0.4549\end{array}$}} \\
\hline$K_{y}$ & & & \\
\hline \multicolumn{4}{|c|}{ Allocator weighting matrices } \\
\hline $\bar{W}$ & \multirow{2}{*}{\multicolumn{3}{|c|}{ 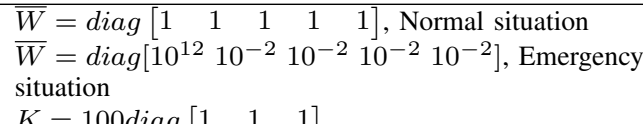 }} \\
\hline$K$ & & & \\
\hline
\end{tabular}
the controller synthesis described in Section V. The allocator weighting matrices $\bar{W}$ and $K$ chosen for this test are presented
Table I: Vehicle, trajectory, controller and allocator parameters
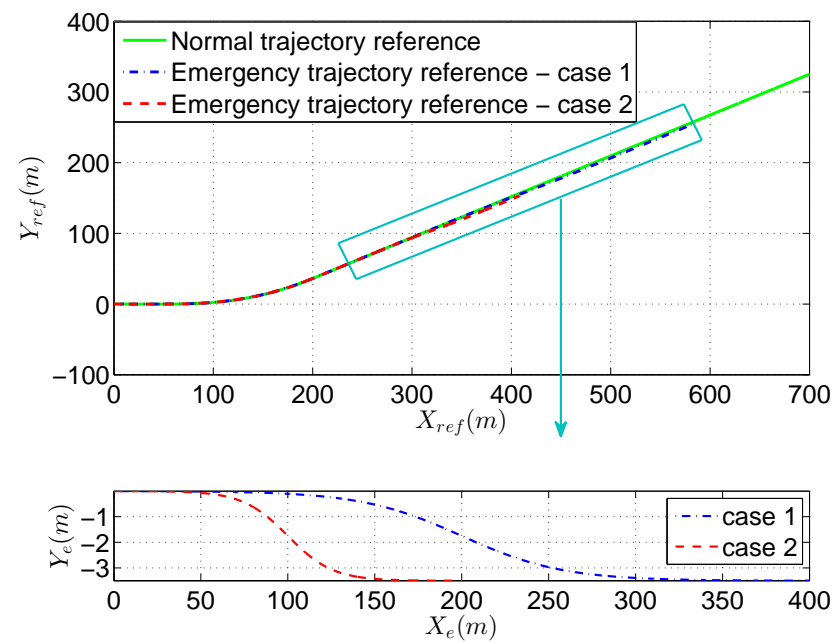

Figure 13: Reference trajectory

in Table I. The weighting matrix $\bar{W}$ changes according to the FDI information. Effectively, in normal driving situation, it is chosen so that none of the actuator action is penalized nor promoted with respect to the others. Conversely, in emergency condition, the weight of the steering angle component is increased and those acting on the brakes are lessened. The weighting matrix $K$ is chosen so that the allocator speed is sufficient.

\section{B. Test results}

The results described below are valid for the two considered cases (case 1 and case 2). When the steering system is free of fault $(t<8 s)$, the lateral guidance controller output $y_{c}$ and the allocation output $u$ coincide (Fig.14) and to maintain a constant vehicle speed, no braking torques are needed as shown in Figs.15-16. The control system response drastically changes after fault detection occurs at $t=8 \mathrm{~s}$. The so designed allocator sends the steering commands to zero (since unusable 
$\delta_{a}=0$ ), solid line in Fig 17, and compensates with differential braking action as in Figs.15-16 to obtain the same $y_{c}$ effect.

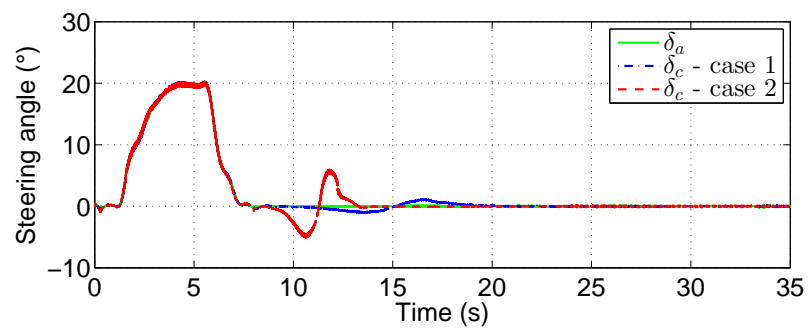

Figure 14: Steering angle time response

In fact, the differential braking aims to turn the vehicle and ensures the lateral guidance toward a safe stop. An accurate results analysis shows, as a matter of fact, that a right turn is obtained by null steering angle $\delta_{a}$ and a higher braking torque on the rear right wheel $T_{b_{r r}}$. In the same way, when the vehicle has to turn left, the steering signal $\delta_{c}$ is mainly replaced by rear left torque $T_{b_{r l}}$ as shown in Figs.15-16.
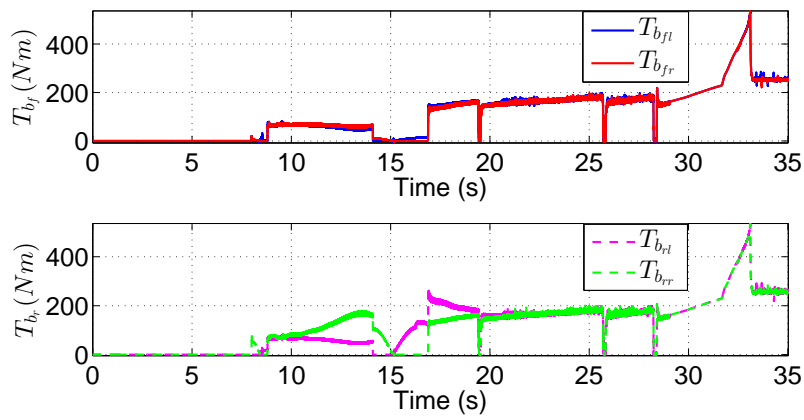

Figure 15: Braking torques - case 1
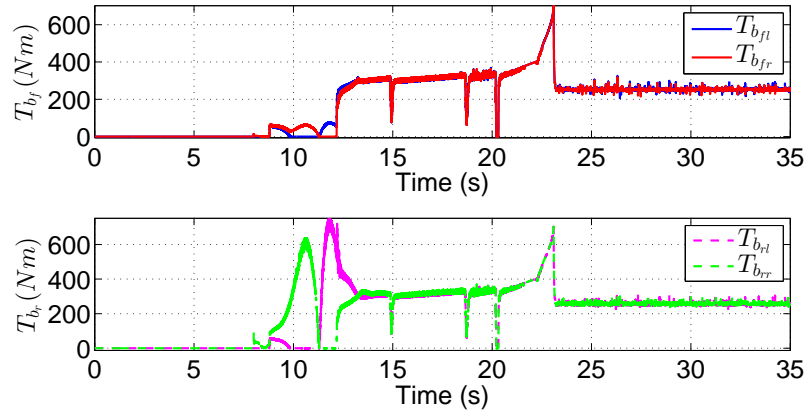

Figure 16: Braking torques - case 2

Unsurprisingly, the differential braking action strongly affects the longitudinal motion of the vehicle as soon as the steering fault appears as can be seen in Fig.17. When the vehicle reaches the stop lane, the lateral action is no longer required $\left(\delta_{c}=0\right)$, and the four braking commands returns to a symmetric behavior $\left(T_{b_{i}}=T_{w b}^{*}\right)$. This brings the vehicle to a full stop based on the computed guidance reference speed profile.

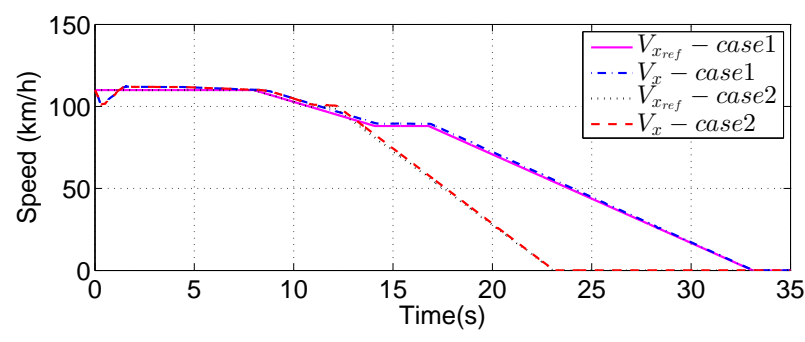

Figure 17: Speed tracking

The proposed approach offers good guidance performances that can be checked through the admissible tracking errors $\left(e_{y} \leq 20 \mathrm{~cm}\right.$ and $\left.e_{\psi} \leq 1^{\circ}\right)$ as shown in Fig.18.
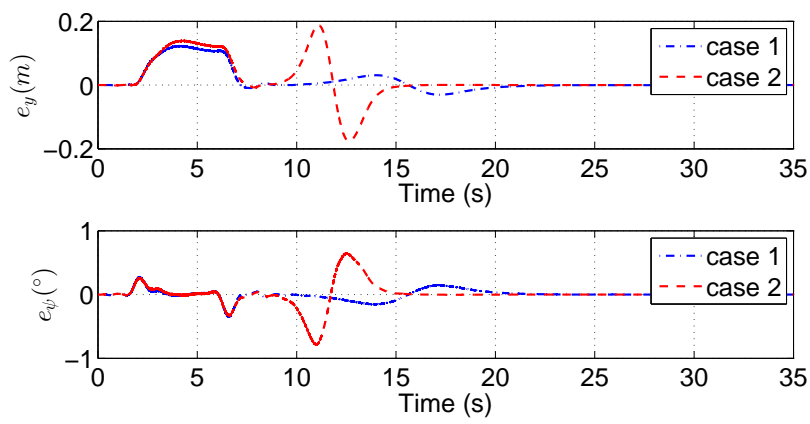

Figure 18: Tracking errors

\section{Results discussion}

Analysis of the two test cases reported above demonstrates the consistency of the implemented control architecture. In detail, it can be noticed that a longer $d_{\text {Long }}$ and a consequently smaller $\rho_{\max }$ (case 1) imply a smaller lateral control command $\delta_{c}$, which reflects in less activity on the brakes during emergency situation. Vice-versa, in case 2 higher braking torque values can be detected (Fig.15-16). In addition, the speed profile decelerates faster in case 2 to reach a quicker stop to meet the smaller $d_{\text {Long }}$ constraint.

It is suitable to consider larger $d_{\text {Long }}$ when no obstacle is detected in front of the vehicle, and thus smaller $\rho_{\max }$, since it requires less braking energy. This remark is related to the fact that tires saturation due to strong braking leading to vehicle lateral instability is not treated in this study.

\section{CONCLUSION}

This paper presents a hierarchical guidance control architecture for the vehicle behavior handling during an emergency situation (steering system failure). To compose this architecture, a reference trajectory and a speed profile for the emergency situation are proposed. These references allow the vehicle to reach safely the emergency stop strip. Then, a guidance controller, composed of a lateral and a longitudinal controller, ensures the references tracking by providing the appropriate control inputs, namely the steering angle and the four wheel brakes. A control allocation is finally developed 
to manage the control inputs for both the normal and the emergency situations. Its role is crucial in this study because it penalizes the steering system, when the latter is defective, and replaces it by differential braking to keep ensuring the lateral guidance. The influence of the choice of the emergency trajectory parameters on the emergency guidance is discussed.

This approach, being based on differential braking, requires a certain minimum speed to guide the vehicle in the emergency situation since the differential braking strongly affects the longitudinal dynamics which can cause the vehicle to stop in the middle of the lane change maneuver. In addition, the possible tires saturation due to the differential braking is not treated here. The partial efficiency loss of the steering system could also be considered by acting on the weighting matrices of the allocator.

\section{REFERENCES}

[1] E. Thorn, S. Kimmel, and M. Chaka, "A framework for automated driving system testable cases and scenarios," Department of Transportation, NHTSA, Tech. Rep DOT HS 812 623, 2018.

[2] A. Eskandarian, Handbook of Intelligent Vehicles, 1st ed., ser. Automotive Engineering. Springer, 2012.

[3] Y. S. Son, W. Kim, S. Lee, and C. C. Chung, "Robust multirate control scheme with predictive virtual lanes for lane-keeping system of autonomous highway driving," IEEE Transactions on Vehicular Technology, vol. 64, no. 8, pp. 3378-3391, 2015.

[4] S. Lee, Y. Son, and C. Chung, "Robust real-time optimal autonomous highway driving control system: Development and implementation," in 19th IFAC World Congress, Cape Town, South Africa, 2014.

[5] D. Watzenig and M. Horn, Automated Driving: Safer and More Efficient Future Driving, 1st ed., ser. Automotive Engineering. Springer, 2017.

[6] V. Milanés, S. E. Shladover, J. Spring, C. Nowakowski, H. Kawazoe, and M. Nakamura, "Cooperative adaptive cruise control in real traffic situations," IEEE Transactions on Intelligent Transportation Systems, vol. 15, no. 1, pp. 296-305, 2014.

[7] R. Rajamani, Vehicle dynamics and control, 2nd ed., ser. Mechanical engineering series. Springer, 2012.

[8] J. Funke and J. C. Gerdes, "Simple clothoid lane change trajectories for automated vehicles incorporating friction constraints," ASME Dynamic Systems, Measurement, and Control, vol. 138, no. 2, pp. 021 002-9, 2015.

[9] K. Liu, J. Gong, A. Kurt, H. Chen, and U. Ozguner, "Dynamic modeling and control of high-speed automated vehicles for lane change maneuver," IEEE Transactions on Intelligent Vehicles, vol. 3, no. 3, pp. 329-339, 2018.

[10] R. Attia, R. Orjuela, and M. Basset, "Combined longitudinal and lateral control for automated vehicle guidance," Vehicle Systems Dynamics, vol. 51, no. 2, pp. 261-279, 2014.

[11] H. Laghmara, M. Boudali, T. Laurain, J. Ledy, R. Orjuela, J.-P. Lauffenburger, and M. Basset, "Obstacle Avoidance, Path Planning and Control for Autonomous Vehicles," in 30th IEEE Intelligent Vehicles, Paris, France, 2019.

[12] D. Gonzalez, J. Perez, V. Milanes, and F. Nashashibi, "A Review of Motion Planning Techniques for Automated Vehicles," IEEE Transactions on Intelligent Transportation Systems, vol. 17, no. 4, pp. 1135-1145, 2015.

[13] J. Funke, M. Brown, S. M. Erlien, and J. C. Gerdes, "Collision Avoidance and Stabilization for Autonomous Vehicles in Emergency Scenarios," IEEE Transactions on Control Systems Technology, vol. 25, no. 4, pp. 1204-1216, 2017.

[14] J. Daniel, G. Pouly, A. Birouche, J.-P. Lauffenburger, and M. Basset, "Navigation-based speed profile generation for an open road speed assistant," in 12th IFAC Symposium on Transportation Systems, Redondo Beach, CA, USA, 2009.

[15] S. Liu, "An on-line reference-trajectory generator for smooth motion of impulse-controlled industrial manipulators," in 7th IEEE International Workshop on Advanced Motion Control. Proceedings, Maribor, Slovenia, 2002.
[16] D. Gonzalez, V. Milanes, J. Perez, and F. Nashashibi, "Speed profile generation based on quintic Bézier curves for enhanced passenger comfort," in 19th IEEE International Conference on Intelligent Transportation Systems (ITSC), Rio de Janeiro, Brazil, 2016.

[17] P. Falcone, F. Borrelli, J. Asgari, H. Tseng, and D. Hrovat, "Predictive Active Steering Control for Autonomous Vehicle Systems," IEEE Transactions on Control Systems Technology, vol. 15, no. 3, pp. 566$580,2007$.

[18] P. Song, C. Zong, and M. Tomizuka, "Combined longitudinal and lateral control for automated lane guidance of full drive-by-wire vehicles," SAE International Journal of Passenger Cars-Electronic and Electrical Systems, vol. 8, no. 2, pp. 419-424, 2015.

[19] H. Luu, L. Nouveliere, and S. Mammar, "Dynamic programming for fuel consumption optimization on light vehicle," in 6th IFAC Symposium on Advances in Automotive Control, Munich, Germany, 2010.

[20] P. Shakouri, A. Ordys, D. Laila, and M. Askari, "Adaptive cruise control system: Comparing gain-scheduling PI and LQ controllers," in 18th IFAC World Congress, Milano, Italy, 2011.

[21] R. Marino, S. Scalzi, and M. Netto, "Nested PID steering control for lane keeping in autonomous vehicles," Control Engineering Practice, vol. 19, no. 12, pp. 1459-1467, 2011.

[22] N. Minoiu Enache, M. Netto, S. Mammar, and B. Lusetti, "Driver steering assistance for lane departure avoidance," Control Engineering Practice, vol. 17, no. 6, pp. 642-651, 2009.

[23] M. Boudali, O. Orjuela, and M. Basset, "A comparison of two guidance strategies for autonomous vehicles," in 20th IFAC World Congress, Toulouse, France, 2017

[24] L. Svensson, L. Masson, N. Mohan, E. Ward, A. P. Brenden, L. Feng, and M. Torngren, "Safe Stop Trajectory Planning for Highly Automated Vehicles: An Optimal Control Problem Formulation," in 29th IEEE Intelligent Vehicles Symposium, Changshu, China, 2018

[25] M. Boudali, O. Orjuela, M. Basset, and R. Attia, "Emergency autonomous vehicle guidance under steering loss," in 29th IEEE Intelligent Vehicles Symposium, Changshu, China, 2018.

[26] J. Lee, H.Lee, J.Kim, and J.Jeong, "Model-based fault detection and isolation for electric power steering system," in International Conference on Control, Automation and Systems, Seoul, Korea, 2007.

[27] S. A. Arogeti, D. Wang, C. B. Low, and M. Yu, "Fault detection isolation and estimation in a vehicle steering system," IEEE Transactions on Industrial Electronics, vol. 59, no. 12, pp. 4810-4820, 2012.

[28] W. Garrott, M. Heitz, and B. Bean, "Experimental measurement of the stopping performance of a tractor-semitrailer from multiple speeds," Department of Transportation, NHTSA, Tech. Rep DOT HS 811 488, 2011.

[29] N. Kapania and J. C. Gerdes, "Design of a feedback-feedforward steering controller for accurate path tracking and stability at the limits of handling," Vehicle System Dynamics, vol. 53, no. 12, pp. 1687-1704, 2015.

[30] R. Orjuela, D. Ichalal, B. Marx, D. Maquin, and J. Ragot, "Chapter 9 - polytopic models for observer and fault-tolerant control designs," in New Trends in Observer-Based Control, ser. Emerging Methodologies and Applications in Modelling, O. Boubaker, Q. Zhu, M. Mahmoud, J. Ragot, H. Karimi, and J. Dávila, Eds. Academic Press, 2019, pp. $295-335$.

[31] L. Nouvelière and S. Mammar, "Experimental vehicle longitudinal control using a second order sliding mode technique," Control Engineering Practice, vol. 15, no. 8, pp. 943 - 954, 2007.

[32] L. Zaccarian, "Dynamic allocation for input redundant control systems," Automatica, vol. 45, no. 6, pp. 1431-1438, 2009.

Faïza Khelladi obtained a B.E.degree in avionics from the aeronautic and aerospace institute, Blida (Algeria) in 2014 and an M.Sc. in automatic control, mechatronic, automobile, aeronautic \& aerospace from the University of Bordeaux (France) in 2017. Currently she is pursuing her Ph.D. in control theory at the University of Haute-Alsace, France. Her areas of interest are control architectures design for autonomous vehicle guidance and stabilization using control allocation techniques. 
Mohamed Boudali obtained an engineering degree in automatic control from l'Ecole polytechnique d'Alger (Algeria) in 2013, and an M.Sc. degree in automatic control from l'Ecole Centrale de Nantes (France) in 2014. In 2019, he received a Ph.D. degree in automatic control from the University of HauteAlsace, France. His areas of interest are design of control architectures for autonomous vehicle guidance. Currently, he is an ADAS engineer.

Rodolfo Orjuela received an M.Sc. in artificial intelligence, pattern recognition and robotics from the University of Paul Sabatier (France) in 2004, and an M.Sc. in automatic control from the University of Bordeaux (France) in 2005. In 2008, he received a Ph.D. degree in automatic control and signal processing from the National Polytechnic Institute of Lorraine (France). Since 2009, he has been as assistant professor of automatic control at the University of Haute-Alsace (France) with the IRIMAS Laboratory. His research interests include design of hierarchical control laws applied to automated guidance of on-ground vehicles (automotive and aircraft). Control law reconfiguration and fault tolerant control are also of interest for him. These works are mainly based-on predictive control, control allocation, linear parameter varying systems and multiple-models. He is a member of the IFAC Technical Committee 7.5 on Intelligent Autonomous Vehicles.

Mario Cassaro received the B.E. degree in aerospace engineering from the University of Palermo, Palermo, Italy, in 2008, and the M.Tech. and Ph.D. degrees in aerospace engineering from the Polytechnic of Turin, Turin, Italy, in 2011 and 2015, respectively. In 2013, he joined the Department of Aerospace Engineering, Clarkson University, Postdam, New York, USA, as Adjunct Instructor. Since October 2016, he has been with the DTIS Département traitement de l'information et systems, IDCO Identification Dynamics and Control, ONERA Toulouse, France, where he was a post-doc researcher until 2018, and became a Research Scientist in 2018. His main research interests include aircraft and rotary wing system modeling, simulation and control, autonomous system design, nonlinear aeroelastic phenomena control, nonlinear adaptive and robust control, data fusion and control allocation.

Michel Basset received the Ph.D. degree in automatic control from the University of Haute-Alsace (France) in 1991 and the next year, as assistant professor, he joined the MIPS now IRIMAS laboratory to continue his research works in experimental modelling and control activities but with applications in the automotive and aeronautical domains. In 2003, he was appointed a senior lecturer and in 2005 a full professor in the domain of control engineering and signal processing at the University of Haute-Alsace. His research interests focus on on-ground vehicle modelling and in particular tyre-road interface, experimental identification, analysis and control with a particular focus on robust control, model predictive control and control allocation. They lead to vehicle behaviour characterization and the development of embedded systems as ADAS and automated controls for connected autonomous vehicle. He is the author or co-author of numerous papers, book chapters, near 18 patents and teaching documents. He also gives courses on automatic control and signal processing at ENSISA engineering school in Mulhouse. He also an active member of the IFAC Technical Committees 7.1. and 7.5 on Automotive Control.

Clément Roos graduated from ISAE-SUPAERO (The French Superior Institute of Aeronautics and Space) in 2004. He received the Ph.D. degree in Automatic Control from ISAE-SUPAERO in 2007, and the French habilitation degree from University Paul Sabatier, Toulouse, in 2018. Since 2007, he has been with ONERA The French Aerospace Lab, where he is now a Senior Scientist in the Information Processing and Systems Department. His research interests focus on aerospace systems modeling, analysis and control, with a particular focus on robustness analysis, robust control, anti-windup design and control allocation. He is the author or co-author of several papers, book chapters, teaching documents and Matlab toolboxes. He also gives courses on automatic control, signal processing and flight mechanics at ISAE-SUPAERO, where he is recognized as a Professor (ERE distinction). 